\section{Correlation of structure and function of the macula in patients with retinitis pigmentosa}

\author{
Abstract \\ Purpose To correlate the structure of the \\ macula, as measured by spectral-domain \\ optical coherence tomography (SD-OCT) and \\ function, as measured by microperimetry \\ (MAIA) in patients with retinitis pigmentosa \\ (RP) and relatively good visual acuity. \\ Design Prospective, cross-sectional, non- \\ intervention study. \\ Subjects Patients with RP. \\ Methods Thirty patients with RP and good \\ central visual acuity were identified. Each \\ patient underwent SD-OCT of the macula \\ and microperimetry. The images were \\ overlaid using the custom-designed \\ software. The retinal sensitivity by \\ microperimetry was correlated with \\ corresponding retinal thickness, as \\ measured by the SD-OCT. ELM, COST, and \\ IS/OS junction were scored as intact, \\ disrupted, or absent. \\ Main outcome measures Comparing the \\ retinal sensitivity on the MAIA with various \\ measurements on the SD-OCT. \\ Results The retinal sensitivity on the \\ MAIA showed a significant correlation \\ with total retinal thickness and outer \\ retinal thickness on the SD-OCT. There \\ was no association with either the inner \\ retinal thickness or the choroidal thickness. \\ ORT showed a statistically significant \\ correlation with the anatomical classifica- \\ tion of ELM $(r=-0.76, P<0.001)$, IS/OS \\ $(r=-0.800, P<0.001)$, and COST $(r=-0.733$, \\ $\mathbf{P}<0.001)$. \\ Conclusion This study determined that there \\ was a high correlation of the structure and \\ function of the central macula in patients \\ with RP. These studies are important to \\ establish surrogate markers that can be used \\ as end points for various tests in future \\ therapeutic clinical trials.
}

R Battu', A Khanna', B Hegde², TTJM Berendschot ${ }^{3}$, S Grover ${ }^{4}$ and JSAG Schouten ${ }^{3}$

Eye (2015) 29, 895-901; doi:10.1038/eye.2015.61; published online 8 May 2015

\section{Introduction}

Retinitis pigmentosa (RP) is the most common type of inherited retinal degenerative disease characterized by loss of photoreceptors, night blindness, bone spicule-like pigmentary changes in the midperiphery and decreased or undetectable responses on an electroretinogram. ${ }^{1,2} \mathrm{RP}$ is a spectrum of diseases characterized by progressive peripheral visual field constriction that can eventually involve the macula with loss of central visual acuity. There is no effective intervention, although several clinical trials are underway to either slow down or arrest the progression of the disease. ${ }^{3-7}$ Progression in RP has traditionally been monitored in the past by assessing visual fields and full-field electroretinography. Although Goldmann visual field testing was the gold standard, more recently static perimetry using the Humphrey Field Analyzer (HFA) (programs 30-2 and 10-2) has been used for this purpose. With the advent of spectral-domain optical coherence tomography (SD-OCT), it has become standard practice to monitor the progression in the structure of the retina, more specifically, the outer retina.

Microperimetry, or fundus-related perimetry, is a technique that involves visual field testing while observing the retina simultaneously. This allows observation of the point on the retina that is being stimulated. This is an especially useful method to assess visual fields in patients with unstable or eccentric fixation caused by macular pathologies. ${ }^{8}$ Literature suggests that retinal sensitivity, and not visual acuity, is related to quality of life and has shown to be sensitive to
${ }^{1}$ Department of Vitreoretina, Narayana Nethralaya, Bangalore, India

${ }^{2}$ Forus Health Pvt. Ltd, Bangalore, India

${ }^{3}$ University Maastricht, Maastricht, The Netherlands

${ }^{4}$ University of Florida, Jacksonville, FL, USA

Correspondence: R Battu, Department of Vitreoretina, Narayana Nethralaya, 121/C, Chord Road, Rajajinagar, Ist ' $R$ ' Block, Bangalore 560010, India

Tel: +91 80 66121400-04,

Fax: +918023377329.

E-mail: rajanibattu@ gmail.com

Received: 30 September 2014

Accepted in revised form:

4 March 2015

Published online:

8 May 2015 +91 9900265081; 
change of function in a trial in RP patients in contrast to HFA $10-2$. $^{9-11}$

SD-OCT can show cross-sectional images of the retina with an axial resolution of up to $5 \mu \mathrm{m} .{ }^{12}$ This resolution enhances visualization of the various retinal layers, particularly at the level of the external limiting membrane (ELM) and photoreceptor inner segment/outer segment (IS/OS) junction. ${ }^{13}$

Several studies have highlighted the importance of the IS/OS layer integrity in studying the progression of RP. ${ }^{13-15}$ Studies have also shown the gradual reduction in the IS/OS layer assessed by the fundus autofluorescence (FAF) ring. ${ }^{16}$

In a study in 2011, Lupo et al ${ }^{17}$ studied the relation between SD-OCT and microperimetry in RP. Lenassi et al investigated the ultrastructural details on the SD-OCT and compared them with the function of the macula, as measured by the microperimetry in these patients. ${ }^{18}$ The purpose of the current study was to perform a structurefunction correlation of the retinal sensitivity on the MAIA to thickness of retinal layers and structural changes in the IS/OS, COST layer, and ELM on the SD-OCT.

\section{Materials and methods}

The study was a prospective case series, approved by the ethics committee of Narayana Nethralaya, Bangalore, India. All the patients consented for the study, and for publication of their data and procedures adhered to the tenets of Declaration of Helsinki. Thirty-four eyes of 34 patients diagnosed with non-syndromic RP based on clinical examination, visual field measurements, and electrophysiology were enrolled into the study. The visual acuity in each eye was measured using the Snellen's visual acuity chart. The eye with the better central visual acuity was selected; if both were the same, the right eye was selected. Patients with vision less than $6 / 36$ on the Snellen chart, macular lesions, or macular edema on fundus examination or SD-OCT were excluded to ensure a stable fixation during microperimetry and to exclude lower microperimetry values due to macular edema. Thirty eyes were used for statistical analysis.

All patients underwent microperimetry on the MAIA (CenterVue, Padova, Italy) using a predefined pattern that covered the central $10^{\circ}$. The Macular Integrity Assessment or MAIA microperimeter uses a scanning laser to perform retinal imaging and an LED light as a stimulus. The eye tracking system on the MAIA was used to ascertain a stable fixation during the entire study. Points corresponding to the foveal center and the horizontal and vertical lines were used for statistical analysis. Patients who could not maintain a steady fixation underwent a repeat testing, and the second test was used for analysis if fixation was stable. Fixation was considered stable if the reliability index was $>60 \%$. If there was no fixation in the fovea, patients were excluded.

SD-OCT was performed with the Spectralis (Heidelberg Engineering, Heidelberg, Germany). The simultaneous confocal scanning laser ophthalmoscope (cSLO) on the Spectralis allowed precise localization of the anatomical landmarks. The eye tracking system ensured a stable fixation during the entire test. Multiple single scans were averaged at the same location to obtain an increased signal-to-noise ratio. A 100-ART vertical and horizontal scan centered on the fovea was obtained on the SD-OCT for each patient within an area of central $30^{\circ}$. Enhanceddepth imaging scans were obtained to assess the choroidal thickness.

An overlay was prepared using a customized software. The MAIA images were overlaid on the cSLO images of the OCT and matched using blood vessels as a coregistration marker. Structural details on the SD-OCT corresponding to the retinal sensitivity at the central (foveal), $1^{\circ}, 3^{\circ}$ and $5^{\circ}$ horizontal and vertical lines on the MAIA were compared (Figure 1).

The inner and outer retinal thickness and the choroidal thickness on the SD-OCT were assessed at each point corresponding to the central and six horizontal and vertical points within the central $10^{\circ}$ on the MAIA. Two observers (RB, AK) made the measurements independent of each other. The mean values were calculated and used
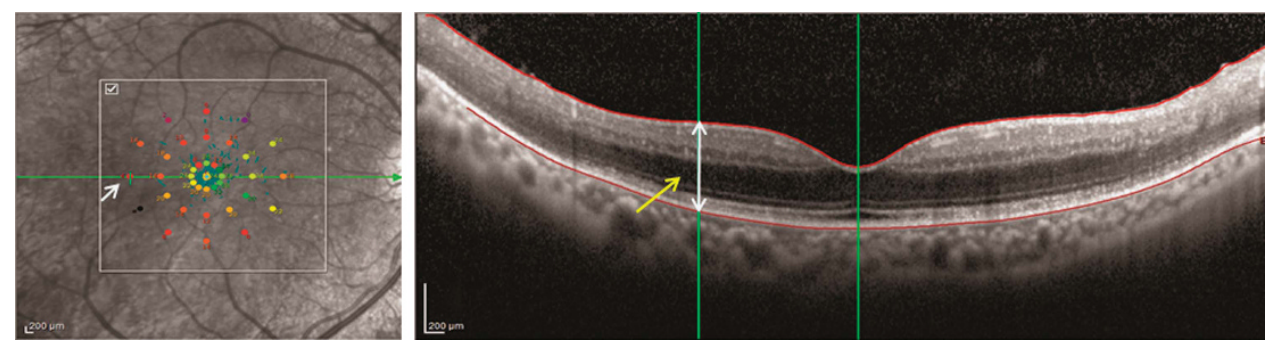

Figure 1 The MAIA of the right eye of a subject overlaid on the cSLO image of the SD-OCT (100 ART horizontal scan) with coregistration of the blood vessels using a customized software. The point measured corresponds to the $5^{\circ}$ temporal location on the MAIA (white arrow) and the corresponding measurement on the SD-OCT (yellow arrow). 
in the analyses. In case there was a difference of $20 \mu \mathrm{m}$ or more between the two observers, the patient was excluded $(n=4)$. On the SD-OCT the ELM, COST layer and IS/OS were assessed and classified as intact, interrupted or absent according to the methods used by others. ${ }^{18}$

The outer retinal thickness (ORT) was measured between the outer border of the retinal pigment epithelium and the inner border of the outer plexiform layer (OPL), to include the inner segments, outer segments and the outer nuclear layer; the inner retinal thickness (IRT) was measured between the vitreoretinal interface and the inner border of the OPL. ${ }^{19}$ Submacular choroidal thickness (CT) was measured manually from the inner border of the sclera to the outer border of the RPE vertically using the calipers of the Heidelberg reader software. ${ }^{20}$

\section{Statistical analysis}

For statistical analysis Snellen visual acuity was converted to LogMAR units. To determine the relation between retinal sensitivity on the microperimetry and the retinal thickness at corresponding locations on the SD-OCT, the IRT, ORT, the choroidal thickness, ELM, IS/OS and COST abnormalities at different eccentricities were measured. A linear mixed models (LMM) analysis was performed with subject ID as a grouping factor, retinal sensitivity as an independent variable, and SD-OCT values, eccentricity, and site (foveal, inferior, superior, nasal, and temporal) as covariates, as well as their interaction terms. All data were analyzed using the statistical software package SPSS version 22.0 (IBM Corporation, New York City, NY, USA).

\section{Results}

Thirty eyes of 30 patients were studied, 23 males and 7 females. Mean age was $32 \pm 12$ years (range 12-63) and mean $\log M A R$ visual acuity was $0.19 \pm 0.17$. There was no statistical difference between males and females either in age or LogMAR visual acuity.

On the MAIA, the average reliability index was $94.23 \%$ (range 67-100\%). The average duration to perform the test was 6'52" (range $\left.4^{\prime} 12^{\prime \prime}-18^{\prime} 51^{\prime \prime}\right)$. The average macular integrity was 90.35 (range 44.3-100). The average threshold was $17.58 \mathrm{~dB}$ (range 2.8-30.1 dB). The mean fixation stabilities were $77.63 \%$ (P1) and $89.80 \%$ (P2). The fixation plot showed that the average bivariate contour ellipse area at $63 \%$ was $2.41^{02}$ and at $95 \%$ was $14.55^{\circ} .^{2}$ There was a negative correlation between LogMAR values and sensitivity at the fovea $(r=-0.52, P=0.004)$; the lower the visual acuity, the worse was the retinal sensitivity.

On the SD-OCT, the mean total foveal thickness was $211 \mu \mathrm{m}$ (range 97-281 $\mu \mathrm{m}$ ), the average inner retinal thickness being $122 \mu \mathrm{m}$ (range $62-201 \mu \mathrm{m}$ ) and outer

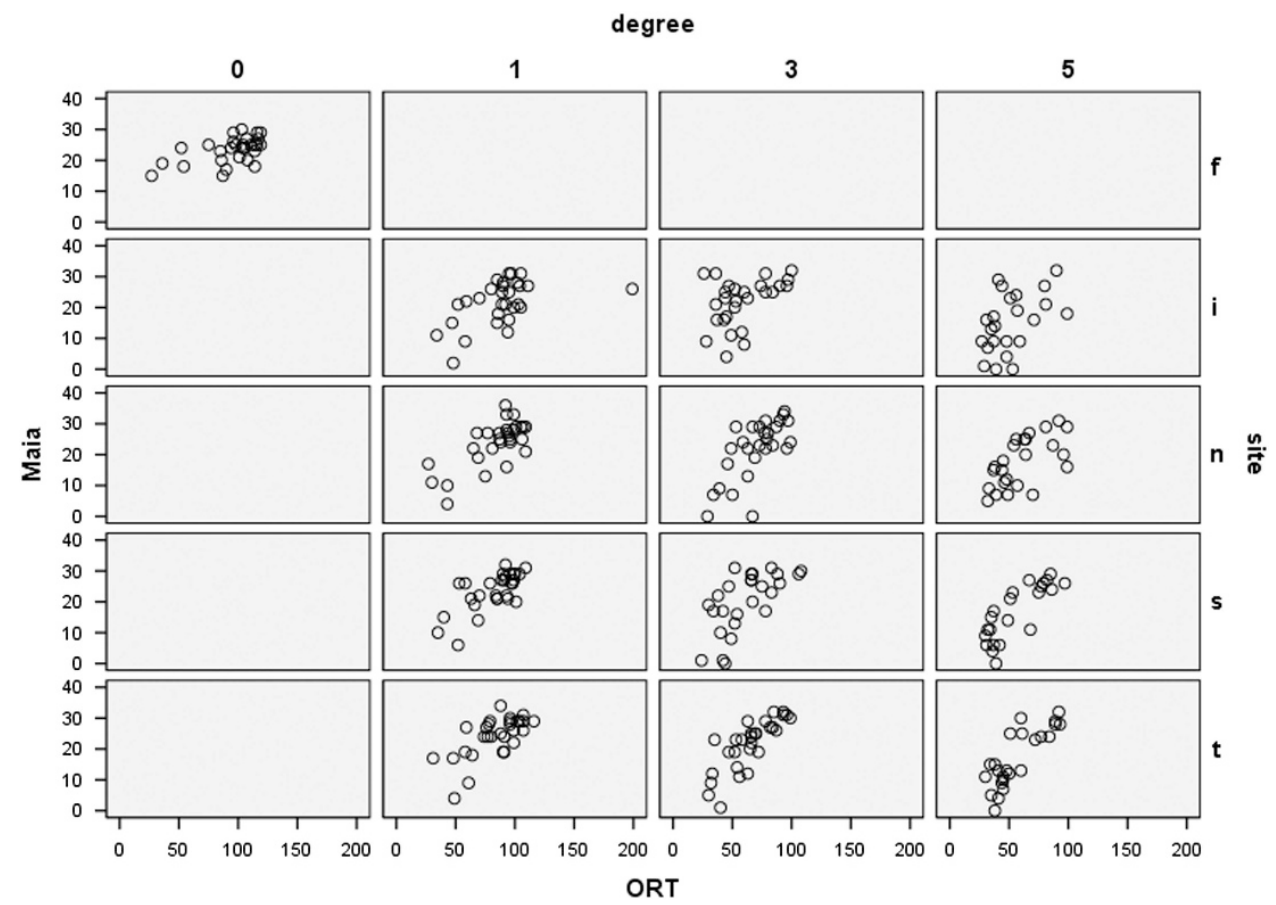

Figure 2 Scatter plot showing the relation between MAIA values of ORT for the different sites (f-fovea, i-inferior, n-nasal, s-superior, $\mathrm{t}$ - temporal) shown in different rows and eccentricities (columns). The correlation coefficients included are all significant $(P<0.005)$. 
retinal thickness being $93 \mu \mathrm{m}$ (range $27-119 \mu \mathrm{m}$ ). The mean choroidal thickness in the subfoveal area was $232 \mu \mathrm{m}$ (range $83-439 \mu \mathrm{m}$ ).

Figure 2 shows the relation between retinal sensitivity and ORT; for all different sites and eccentricities we found a significant correlation. Figure 3 shows the mean ORT for different sites and eccentricities. LMM analyses revealed a significant association between retinal sensitivity and ORT. This association was different for the different sites and eccentricities, that is, showing significant interaction terms.

ELM, COST, and IS/OS junction were scored as intact, disrupted or absent. Analysis of retinal sensitivities by location yielded us a total of 390 locations. We found that the ELM was intact in 206, disrupted in 74, and absent in 110 locations.

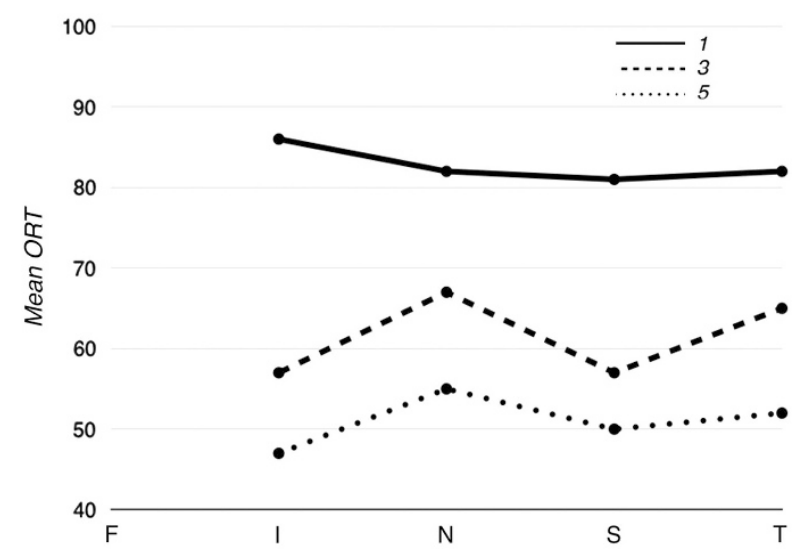

Figure 3 Mean ORT for the different sites. Different lines represent different eccentricities. Similar graphs were drawn for the IRT and EDI (f-fovea, i-inferior, n-nasal, s-superior, t-temporal).
Similar analysis for IS/OS gave 182, 74, and 134, respectively, and analysis of COST gave 88,68 , and 234, respectively. All three layers were intact in 86 , disrupted in 1 , and absent in 90 locations. ORT showed a statistically significant correlation with the anatomical classification of ELM $(r=-0.76, P<0.001)$, IS/OS $(r=-0.800, P<0.001)$, and COST $(r=-0.733, P<0.001)$.

Sensitivity values at different locations and eccentricities were grouped according to the above anatomical integrity classification, and we found highest mean sensitivities in the intact group, lower for the disrupted group, and lowest for the absent group for all three layers (Table 1).

Bivariate correlation showed that MAIA correlated significantly with sex $(P<0.001)$, spherical equivalent $(P<0.001)$, TRT $(P<0.001)$, outer retinal thickness $(P<0.001)$, ELM $(P<0.001)$, IS/OS $(P<0.027)$, COST $(P<0.001)$, location $(P=0.032)$, and eccentricity $(P<0.001)$.

Using the above data we performed a multivariate regression (Table 2) keeping the retinal sensitivity as the dependent variable and age, sex, spherical equivalent, ORT, IRT, TRT, anatomy of the ELM, IS/OS, and COST, and location and eccentricity of measurement. We found that sex, ORT, and the anatomical integrity of ELM and IS/OS had an important role in the retinal sensitivity value.

\section{Discussion}

Lupo et $a l^{17}$ studied the relationship between retinal thickness and retinal function in RP. They, however, only compared the central retinal thickness with the retinal sensitivity on the microperimetry and did not look at the structural details. A study by Lenassi et al assessed the

Table 1 Summary of the mean retinal sensitivities using MAIA at different locations and eccentricities grouped by the anatomical status of the outer retinal layers on SD-OCT

\begin{tabular}{|c|c|c|c|c|c|c|c|c|c|c|c|c|c|}
\hline \multirow[t]{2}{*}{ Location } & \multirow[t]{2}{*}{ Eccentricity } & \multicolumn{4}{|c|}{ IS/OS } & \multicolumn{4}{|c|}{ COST } & \multicolumn{4}{|c|}{ ELM } \\
\hline & & Intact & Disrupted & Absent & P-value ${ }^{\mathrm{b}}$ & Intact & Disrupted & Absent & P-value ${ }^{\mathrm{b}}$ & Intact & Disrupted & Absent & P-value ${ }^{\mathrm{b}}$ \\
\hline Fovea & 0 & 24.43 & 17.63 & 23.00 & $<0.001$ & 25.93 & 17.86 & 21.00 & 0.004 & 24.39 & 20.20 & 7.50 & $<0.001$ \\
\hline \multirow[t]{3}{*}{ Inferior } & 1 & 24.48 & 17.17 & 3.63 & $<0.001$ & 26.23 & 20.56 & 12.75 & $<0.001$ & 24.14 & 14.33 & 5.50 & $<0.001$ \\
\hline & 3 & 25.78 & 14.00 & 18.00 & 0.58 & 27.67 & 25.00 & 18.00 & 0.176 & 25.00 & 18.11 & 16.09 & 0.100 \\
\hline & 5 & 24.50 & 21.00 & 7.96 & $<0.001$ & 26.67 & 18.00 & 9.46 & 0.016 & 21.29 & 11.00 & 7.95 & 0.010 \\
\hline \multirow[t]{3}{*}{ Superior } & 1 & 25.78 & 12.60 & 12.50 & $<0.001$ & 27.55 & 23.22 & 16.90 & 0.002 & 25.73 & 15.67 & 10.50 & $<0.001$ \\
\hline & 3 & 28.00 & 18.43 & 10.07 & $<0.001$ & 29.67 & 28.50 & 13.87 & 0.004 & 25.75 & 17.13 & 7.60 & $<0.001$ \\
\hline & 5 & 26.80 & 14.00 & 7.53 & $<0.001$ & 27.00 & 26.00 & 9.77 & 0.007 & 23.67 & 10.00 & 6.35 & $<0.001$ \\
\hline \multirow[t]{3}{*}{ Nasal } & 1 & 26.48 & 12.00 & 8.50 & $<0.001$ & 27.60 & 23.30 & 17.70 & 0.025 & 25.52 & 16.33 & 12.50 & 0.003 \\
\hline & 3 & 26.50 & 6.20 & 14.43 & $<0.001$ & 28.50 & 25.80 & 16.26 & 0.018 & 26.17 & 10.86 & 12.40 & $<0.001$ \\
\hline & 5 & 23.88 & 12.00 & 10.42 & 0.003 & 27.67 & 21.00 & 11.63 & 0.011 & 20.69 & 11.00 & 8.62 & 0.004 \\
\hline \multirow[t]{3}{*}{ Temporal } & 1 & 25.71 & 17.13 & 19.00 & 0.021 & 27.64 & 24.57 & 18.33 & 0.010 & 25.00 & 22.20 & 11.67 & 0.015 \\
\hline & 3 & 26.46 & 20.14 & 14.00 & 0.002 & 30.60 & 27.00 & 16.32 & $<0.010$ & 26.12 & 19.29 & 7.67 & $<0.001$ \\
\hline & 5 & 26.29 & 15.33 & 7.65 & $<0.001$ & 29.67 & 20.33 & 10.67 & 0.004 & 25.38 & 15.33 & 6.94 & $<0.001$ \\
\hline
\end{tabular}

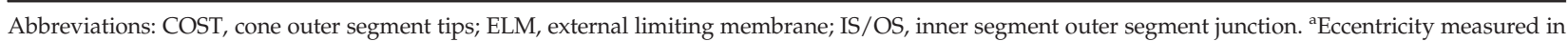
degrees from fovea. ${ }^{b}$ Comparison of means-One-way ANOVA. 
Table 2 Multivariate linear regression model for dependent variable: retinal sensitivity in $\mathrm{db}$ measured with the MAIA (Model $R^{2}=0.607$ )

\begin{tabular}{lccr}
\hline Parameter & Beta & Standardized Beta & P-value \\
\hline Intercept & 25.15 & & $<0.001$ \\
Age (years) & -0.05 & -0.059 & 0.112 \\
Sex (male, female) & $\mathbf{- 4 . 6 4}$ & $\mathbf{- 0 . 1 9 2}$ & $<\mathbf{0 . 0 0 1}$ \\
Sph Eq (in diopters) & -0.162 & -0.042 & 0.251 \\
TRT $(\mu \mathrm{m})$ & -0.050 & -0.266 & 0.542 \\
ORT $(\mu \mathrm{m})$ & $\mathbf{0 . 1 7 5}$ & $\mathbf{0 . 4 6 7}$ & $\mathbf{0 . 0 4 3}$ \\
IRT $(\mu \mathrm{m})$ & 0.070 & 0.372 & 0.393 \\
ELM $^{\text {a }}$ & $\mathbf{- 2 . 9 0 9}$ & $\mathbf{- 0 . 2 4 8}$ & $<\mathbf{0 . 0 0 1}$ \\
ISOS $^{\text {a }}$ & $\mathbf{- 1 . 9 4 2}$ & $-\mathbf{0 . 1 7 0}$ & $\mathbf{0 . 0 0 6}$ \\
COST $^{\text {a }}$ & -0.490 & -0.040 & 0.442 \\
EDI $^{\mathrm{a}}$ & -0.004 & -0.033 & 0.385 \\
Location $^{\mathrm{b}}$ & -0.052 & -0.006 & 0.856 \\
Eccentricity $^{\mathrm{c}}$ & -0.663 & -0.062 & 0.252 \\
\hline
\end{tabular}

Abbreviations: COST, cone outer segment tips; EDI, extended depth imaging of the choroid; ELM, external limiting membrane; IRT, inner retinal thickness; ISOS, inner segment outer segment junction; ORT, outer retinal thickness; Sph Eq, spherical equivalent; TRT, total retinal thickness. antact-1, disrupted-2, absent-3. ' $F$ Fovea-1, temporal-2, nasal-3, superior -4 , inferior $-5 .{ }^{c} 1^{\circ}-1,3^{\circ}-2,5^{\circ}-3$. Bold indicates statistically significant.

correlation of visual function measured by both static and kinetic fundus-related perimetry with structural changes in the inner and outer retina evaluated with SD-OCT and with molecular changes in the RPE visualized with FAF. ${ }^{18}$ Mitamura et $a l^{21}$ found a significant correlation between the length of the IS/OS line and the retinal sensitivity using the MP-1 microperimeter.

Studies to establish the structure-function correlation in retinal degenerative diseases are essential as more and more treatment modalities become available. We developed a custom-made software to assess the structure-function correlation between fundus-related microperimetry and SD-OCT. We found that the retinal sensitivity correlated with the outer retinal structure and an intact ELM and IS/OS junction but not with the inner retinal thickness, choroidal thickness, or the COST layer.

Microperimeters allow for registration of fundus photos with the other imaging modalities and make it possible to compare retinal morphology with visual function. ${ }^{22}$ Retinal sensitivity assessed using microperimetry has been advocated as a potential outcome measure of macular function for clinical trials involving patients with RP. ${ }^{23}$ Cideciyan et al ${ }^{23}$ have established repeatability of microperimetry in patients with RP. In their cohort of patients with ABCA4-associated retinopathy that included RP, they showed that the repeated microperimetry tests showed reasonable concordance and the test-retest variance was not significant. Changes on the SD-OCT have been used in several studies to study the disease progression, ${ }^{13-16}$ and by the same measure may be useful in monitoring response to therapy.

$\mathrm{RP}$ is a heterogeneous group of diseases; mutations in more than 50 genes are known to cause non-syndromic $\mathrm{RP}$ and more than 3100 mutations have been identified in these genes. ${ }^{24,25}$ The gene products involve multiple metabolic pathways localized to mostly rods, or rods and cones, or the retinal pigment epithelium. ${ }^{2}$ These include proteins of rod visual transduction, photoreceptor differentiation, proteins of the cytoskeleton, protein transportation, and retinol metabolism among others. ${ }^{2}$ Mutations in any of the biochemical cycles that alter the phototransduction cascade, including renewal and shedding of the photoreceptor outer segments, visual transduction, and/or retinol metabolism may cause retinitis pigmentosa. ${ }^{26}$

Our study showed that the retinal sensitivity in the macula correlated well with the outer retinal thickness mainly, which included the inner and outer segments of the photoreceptors. It is not surprising that many important biochemical processes occur in the outer retinal layers, and structural preservation of these layers corresponds to a functional preservation.

Issa et $a l^{27}$ showed that in one patient with Usher's syndrome, there was a significant relationship between outer retinal thickness and retinal sensitivity. They demonstrated that good functional preservation was associated with outer retinal thickness $>50 \mu \mathrm{m}$ in spite of extensive disruption of the inner retina.

The study by Lenassi et al ${ }^{18}$ found a high correlation between retinal sensitivity and outer retinal thickness, than overall retinal thickness in patients with RP. We found that the retinal sensitivity correlates well with both outer and total retinal thickness and not inner retinal thickness or the choroidal thickness. Changes in the choroid are probably secondary to changes that occur in the retinal layers, and therefore choroidal thickness may vary in patients with RP.

In addition to the IS/OS line, the integrity of the ELM and COST layers has been described as potential indicators of retinal function; a relation between intact ELM/COST layer and good visual acuity has been described in degenerative eye diseases like age-related macular degeneration, macular hole, and epiretinal membrane. ${ }^{28-31}$

We found that the presence of intact ELM and an intact IS/OS correlated significantly with the retinal sensitivity. Oishi et $a l^{29}$ found a strong correlation between ELM status and visual acuity that was higher than that with the IS/OS in a cohort of patients with age-related macular degeneration (AMD). The status of the ELM was the most important factor for visual acuity. In RP, it is possible that, in addition to the IS/OS, the ELM is a marker of photoreceptor integrity. Progressive degeneration of 
photoreceptors may lead to loss of inner retinal structures, and loss of ELM may indicate a severe degree of damage.

In a similar study, Itoh et al 28,30 found a significant correlation between the presence of an intact COST line and visual recovery in patients who underwent surgery for macular hole. In an earlier study, Srinivasan et $a l^{32}$ had shown that the cone outer segment lengths were greatest in the foveola and decreased with increasing eccentricity. We did not find a significant association between the COST layer and the retinal sensitivity.

Functional mapping of the macula is essential in retinal degenerative diseases. It allows us a window of opportunity to treat before permanent structural damage occurs. It also helps select patients with early functional loss in whom there are better chances of success with an experimental therapy and help efficient monitoring of the effectiveness of any therapy in RP. Although visual acuity is considered to be the standard primary end point in clinical trials of retinal diseases, it is important that other end points be considered in diseases like RP. This study is one such an attempt to identify surrogate end points, which may be useful to monitor future therapies. Sugawara et $a l^{9}$ have established that macular sensitivity determined by microperimetry may be a good predictor of quality of life in RP patients with relatively good vision. Quality of life needs to be an important consideration when future clinical trials and pharmaceutical regulations are considered.

Studies like this help us understand better the structural changes that occur in RP. It would be interesting to study the natural course of the disease and assess the rate of functional loss vis-à-vis the morphological changes in the structure. This may be an important consideration for pharmaceutical interventions. Our study is an attempt to establish the markers that may help identify early structural damage before functional loss is noted. This may further help us understand how soon we need to intervene in the course of the disease for it to prevent irretrievable visual loss. Identifying these markers is important in formulating the inclusion and exclusion criterla for clinical trials in RP.

It is hard to predict whether functional changes precede structural changes in RP. A co-registration of the MAIA and SD-OCT shows the early functional changes associated with micro-structural alterations. Issa et al ${ }^{27}$ suggested that micro-structural alterations precede functional loss, and high-resolution retinal imaging can detect this. If structural changes do precede functional loss, and it is possible to detect these changes on a highdefinition imaging device, this would be of vital importance in patients with RP, as it would mean that therapy can be offered to these patients before they notice a functional visual loss. Adaptive optics may be one such imaging device that may pick up ultrafine structural changes. ${ }^{33}$ A structure function correlation between adaptive optics and microperimetry may be useful to detect the earliest structural changes in RP and other such degenerative retinal disorders.

Our study has limitations. The sample size was small, and it was a cross-sectional study. We did not have the results of genetic studies of these patients.

There is a continuous need to detect degenerative diseases like RP in its earliest stage, when therapy may be most useful and before irreversible damage has occurred. Studies like ours try to identify surrogate end points to monitor response to clinical trials before true functional loss has occurred.

\section{Summary}

\section{What was known before}

- Structure-function correlation in RP. The central retinal thickness on the SD-OCT correlates with the retinal sensitivity on the microperimeter.

- Correlation with outer retinal thickness. Outer retinal thickness correlates with the retinal sensitivity on the OCT.

What this study adds

- In RP, an intact ELM and an intact IS/OS correlated significantly with the visual acuity and retinal sensitivity.

- An intact COST layer does not correlate with the retinal sensitivity.

\section{Conflict of interest}

The authors declare no conflict of interest.

\section{References}

1 Fishman GA. Retinitis pigmentosa. Genetic percentages. Arch Ophthalmol 1978; 96: 822-826.

2 Hamel C. Retinitis pigmentosa. Orphanet J Rare Dis 2006; 1: 40 .

3 Humayun MS, Dorn JD, da Cruz L, Dagnelie G, Sahel JA, Stanga PE et al. Argus II Study Group. Interim results from the international trial of Second Sight's visual prosthesis. Ophthalmology 2012; 119: 779-788.

4 Tan MH, Smith AJ, Pawlyk B, Xu X, Liu X, Bainbridge JB et al. Gene therapy for retinitis pigmentosa and Leber congenital amaurosis caused by defects in AIPL1: effective rescue of mouse models of partial and complete Aipl1 deficiency using AAV2/2 and AAV2/8 vectors. Hum Mol Genet 2009; 18: 2099-2114.

5 Bennett J. Gene therapy for retinitis pigmentosa. Curr Opin Mol Ther 2000; 2: 420-425.

6 Chader GJ, Weiland J, Humayun MS. Artificial vision: needs, functioning, and testing of a retinal electronic prosthesis. Prog Brain Res 2009; 175: 317-332.

7 MacLaren RE, Groppe M, Barnard AR, Cottriall CL, Tolmachova T, Seymour L et al. Retinal gene therapy in patients with choroideremia: initial findings from a phase 1/2 clinical trial. Lancet 2014; 383: 1129-1137. 
8 Rohrschneider KS, Bultmann, Springer C. Use of fundus perimetry (microperimetry) to quantify macular sensitivity. Prog Retin Eye Res 2008; 27: 536-548.

9 Sugawara T, Sato E, Baba T, Hagiwara A, Tawada A, Yamamoto S. Relationship between vision-related quality of life and microperimetry-determined macular sensitivity in patients with retinitis pigmentosa. Jpn J Ophthalmol 2011; 55: 643-646.

10 Sugawara T, Hagiwara A, Hiramatsu A, Ogata K, Mitamura Y, Yamamoto S. Relationship between peripheral visual field loss and vision-related quality of life in patients with retinitis pigmentosa. Eye 2010; 24: 535-539.

11 Tawada A, Sugawara T, Ogata K, Hagiwara A, Yamamoto S. Improvement of central retinal sensitivity six months after topical isopropyl unoprostone in patients with retinitis pigmentosa. Indian J Ophthalmol 2013; 61: 95-99.

12 Alam S, Zawadzki RJ, Choi S, Gerth C, Park SS, Morse L et al. Clinical application of rapid serial fourier-domain optical coherence tomography for macular imaging. Ophthalmology 2006; 113: 1425-1431.

13 Aizawa S, Mitamura Y, Baba T, Hagiwara A, Ogata K, Yamamoto S. Correlation between visual function and photoreceptor inner/outer segment junction in patients with retinitis pigmentosa. Eye 2009; 23: 304-308.

14 Fischer M, Fleischhauer JC, Gillies MC, Sutter FK, Helbig H, Barthelmes D. A new method to monitor visual field defects caused by photoreceptor degeneration by quantitative optical coherence tomography. Invest Ophthalmol Vis Sci 2008; 49: 3617-3621.

15 Jacobson SG, Roman AJ, Aleman TS, Sumaroka A, Herrera W, Windsor EA et al. Normal central retinal function and structure preserved in retinitis pigmentosa. Invest Ophthalmol Vis Sci 2010; 51: 1079-1085.

16 Robson AG, Michaelides M, Saihan Z, Bird AC, Webster AR, Moore AT et al. Functional characteristics of patients with retinal dystrophy that manifest abnormal parafoveal annuli of high density fundus autofluorescence; a review and update. Doc Ophthalmol 2008; 116: 79-89.

17 Lupo S, Grenga PL, Vingolo EM. Fourier-domain optical coherence tomography and microperimetry findings in retinitis pigmentosa. Am J Ophthalmol 2011; 151: 106-111.

18 Lenassi E, Troeger E, Wilke R, Hawlina M. Correlation between macular morphology and sensitivity in patients with retinitis pigmentosa and hyperautofluorescent ring. Invest Ophthalmol Vis Sci 2012; 53: 47-52.

19 Curcio C, Messinger JD, Sloan KR, Mitra A, McGwin G, Spaide RF. Human chorioretinal layer thicknesses measured in macula-wide, high-resolution histologic sections. Invest Ophthalmol Vis Sci 2011; 52: 3943-3954.

20 Dhoot DS, Huo S, Yuan A, Xu D, Srivistava S, Ehlers JP et al. Evaluation of choroidal thickness in retinitis pigmentosa using enhanced depth imaging optical coherence tomography. Br J Ophthalmol 2013; 97: 66-69.

21 Mitamura Y, Aizawa S, Baba T, Hagiwara A, Yamamoto S. Correlation between visual function and photoreceptor inner/outer segment junction in patients with retinitis pigmentosa. Br J Ophthalmol 2009; 93: 126-127.

22 Acton JH, Greenstein VC. Fundus-driven perimetry (microperimetry) compared to conventional static automated perimetry: similarities, differences, and clinical applications. Can J Ophthalmol 2013; 48: 358-363.

23 Cideciyan A, Swider M, Aleman TS, Feuer WJ, Schwartz SB, Russell RC et al. Macular function in macular degenerations: repeatability of microperimetry as a potential outcome measure for ABCA4-associated retinopathy trials. Invest Ophthalmol Vis Sci 2012; 53: 841-852.

24 HGMD, Human Gene Mutation Database (Biobase BiologicalDatabases) 2013. Retrieved from http:/ / www. hgmd.cf.ac.uk/. Accessed June 2014.

25 Retnet, The Retinal Information Network 2013. Retrieved from http://www.sph.uth.tmc.edu/RetNet/. Accessed June 2014. 2014.

26 Wert KJ, Lin JH, Tsang SH. General pathophysiology in retinal degeneration. Dev Ophthalmol 2014; 53: $33-43$.

27 Issa PC, Troeger E, Finger R, Holz FG, Wilke R, Scholl HP. Structure-function correlation of the human central retina. PLoS One 2010; 5: e12864.

28 Itoh Y, Inoue M, Rii T, Hiraoka T, Hirakata A. Significant correlation between visual acuity and recovery of foveal cone microstructures after macular hole surgery. Am J Ophthalmol 2012; 153: 111-119.

29 Oishi A, Hata M, Shimozono M, Mandai M, Nishida A, Kurimoto $\mathrm{Y}$. The significance of external limiting membrane status for visual acuity in age-related macular degeneration. Am J Ophthalmol 2010; 150: 27-32.

30 Itoh Y, Inoue M, Rii T, Hiraoka T, Hirakata A. Correlation between length of foveal cone outer segment tips line defect and visual acuity after macular hole closure. Ophthalmology 2012; 119: 1438-1446.

31 Shimozono M, Oishi A, Hata M, Matsuki T, Ito S, Ishida K et al. The significance of cone outer segment tips as a prognostic factor in epiretinal membrane surgery. Am J Ophthalmol 2012; 153: 698-704

32 Srinivasan VJ, Monsoon BK, Wojtkowski M, Bilonick RA, Gorczynska I, Chen R et al. Characterization of outer retinal morphology with high-speed, ultrahigh-resolution optical coherence tomography. Invest Ophthalmol Vis Sci 2008; 49: 1571-1579.

33 Battu R, Dabir S, Khanna A, Kumar AK, Roy AS. Adaptive optics imaging of the retina. Indian J Ophthalmol 2014; 62: $60-65$. 